\title{
Gamificación y otras estrategias docentes en seminarios y prácticas de laboratorio de la asignatura Nutrición y Bromatología
}

\section{José Miguel HeRnández HIERRO}

Laboratorio de Color y Calidad de Alimentos Departamento de Nutrición y Bromatología, Toxicología y Medicina Legal Universidad de Sevilla

Facultad de Farmacia jmhhierro@us.es

ORCID: https://orcid.org/0000-0002-2329-3024

D.O.I.: http://dx.doi.org/10.12795/JDU.2018.i01.62 Pp.: 1100-1117

\section{Resumen}

Durante el curso 2018/2019 se ha realizado un ciclo de mejora en la asignatura Nutrición y Bromatología en la partes correspondientes a los seminarios y prácticas de laboratorio. La propuesta para este curso se ha centrado en la gamificación de los seminarios para conseguir por parte del alumnado un seguimiento más continuado de esta parte de la asignatura. En el caso de las sesiones de laboratorio, los alumnos han planteado dos preguntas a las que la práctica daba respuesta. 
Tras la realización de la práctica se volvió a pedir otra vez las preguntas y mediante un pequeño debate se decidió si la realización de dicha práctica había dado respuesta o no y con qué conceptos básicos estaban relacionadas las respuestas obtenidas en un mapa de contenidos. Tras dicha actividad se evaluaron los modelos mentales iniciales y finales de los estudiantes.

Palabras clave: Nutrición y Bromatología, Grado en Farmacia, docencia universitaria, innovación docente universitaria, cambio metodológico.

\section{Breve descripción del contexto}

\section{Contexto de la intervención}

La Universidad de Sevilla cuenta en la actualidad con un total de 67147 alumnos, que cursan estudios en 43 centros (Facultades y Escuelas Universitarias) y alrededor de 3000 profesores, que pertenecen a 105 departamentos. El Grado de Farmacia de esta Universidad está estructurado en 4 años y una carga de 240 ETCS distribuida en materias básicas, obligatorias, optativas, prácticas tuteladas y el trabajo fin de Grado. Nutrición y Bromatología es una asignatura de carácter obligatorio del tercer curso de dicha titulación, impartida durante el primer cuatrimestre en la Facultad de Farmacia. Esta Facultad cuenta con aproximadamente 250 profesores con encargo docente agrupados en 12 departamentos (Decanato de la Facultad de Farmacia de Sevilla, 2017). Desde el curso 2012-2013 he participado, con mayor o menor carga de grupos, en la docencia de esta asignatura en la que se plantea la intervención.

Jornadas de Formación e Innovación Docente del Profesorado | № 1 (2018) Esta obra se distribuye con la licencia Creative Commons 


\section{Diseño previo del ciclo de mejora docente}

\section{Descripción de la docencia}

La asignatura Nutrición y Bromatología tiene asignados 7.5 créditos ECTS, que a su vez se encuentran divididos en clases teóricas, prácticas y seminarios (4.5 teóricos, 1.5 prácticos y 1.5 teórico-prácticos).

En primer lugar se presenta un esbozo de los tres pilares que articulan toda la asignatura en la Figura 1.

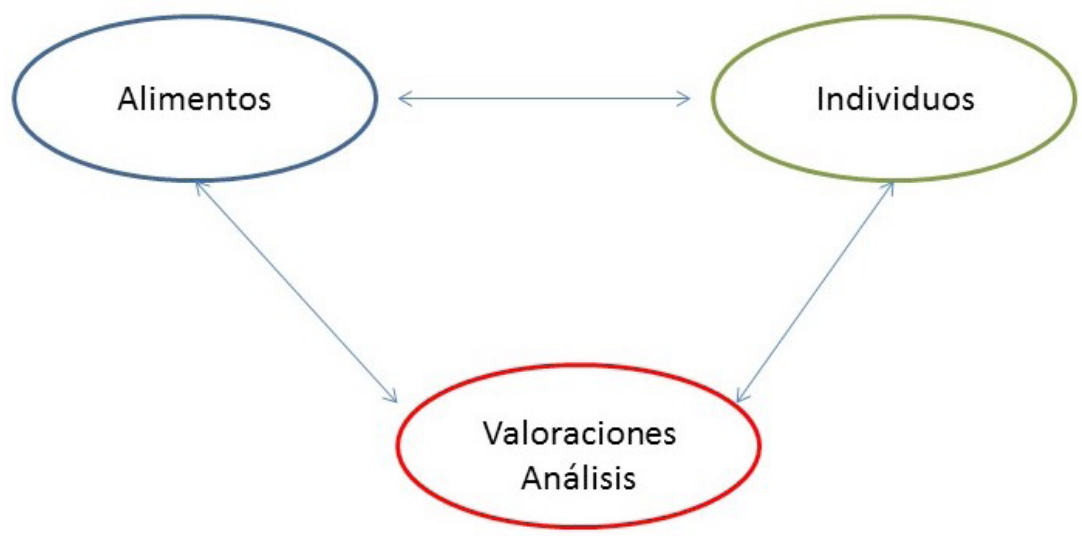

Figura 1. Mapa de contenidos general de la asignatura Nutrición y Bromatología.

La propuesta para el curso pasado se centró en la gamificación de los seminarios para conseguir por parte del alumnado un seguimiento más continuado de esta parte de la asignatura (Hernández Hierro, 2017). Uno de los mayores problemas a la hora de comenzar fue la creación de grupos de trabajo cooperativo de larga duración, que siempre tiene asociado un grado de complejidad elevado, agravado por la falta de información sobre la pertenecía a un grupo u otro de la asignatura por temas administrativos. Otro de los aspectos problemáticos detectados es que hay partes del juego, concretamente el sorteo de puntos, 
que no han tenido buena aceptación y se debería de replantear la utilidad de mantenerlo o sustituirlo por otra estrategia con la misma finalidad. El resultado de la aplicación en el curso anterior fue muy satisfactorio al lograr un buen seguimiento de esa parte de la asignatura y las calificaciones finales de los alumnos resultaron muy buenas. En cuanto al desarrollo del juego, la parte del sorteo de puntos fue muy criticada por lo se ha eliminado esta vez al igual que la creación de grupos de trabajo cooperativo. Se ha utilizado Socrative para facilitar mediante el juego la adquisición de conceptos relativos a esa parte de la asignatura (AulaPlaneta, 2015). La aplicación nace en el 2010 como soporte para las clases como gestor de la participación de los estudiantes en el aula en tiempo real utilizando un amplio abanico de dispositivos móviles. Dicha aplicación permite realizar test, evaluaciones, actividades, etc. y manejar los datos por el docente. Dentro del proceso anteriormente mencionado se necesita de herramientas que puedan ser utilizadas por profesor y estudiante de forma sencilla y eficiente. En el caso del alumnado es primordial que además sea una forma atractiva de uso, como puede ser el propio teléfono móvil al que el alumnado suele estar muy acostumbrado. Se pretendió dar un carácter voluntario pero con repercusión en la calificación de la asignatura. Este aspecto de voluntariedad deberá ser eliminado en futuras intervenciones.

El mapa de contenidos de estos temas se muestra en la Figura 2. En este mapa se puede observar el detalle de los contenidos tratados, preguntas clave y diferenciación entre lo conceptual, procedimental (letras en verde) y actitudinal (letras moradas en cursiva). 


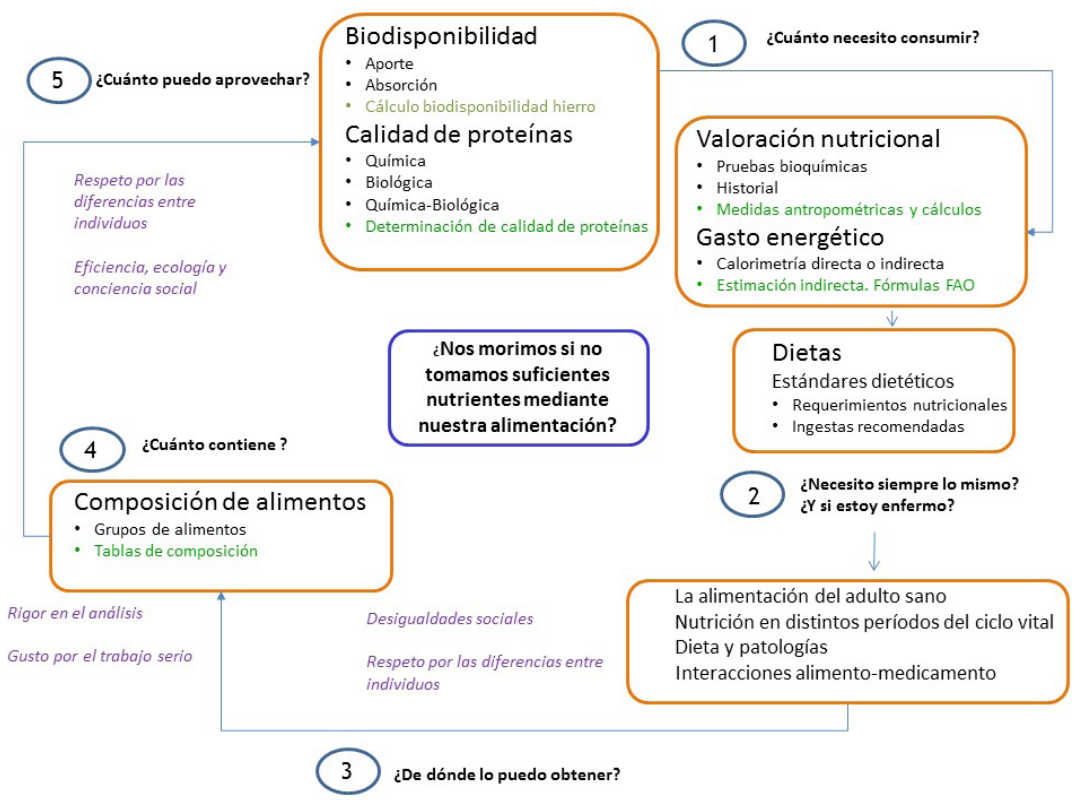

Figura 2. Mapa de contenidos.

\section{Modelos metodológicos}

\section{Modelo metodológico habitual}

El modelo metodológico aplicado tiene cuatro partes que son introducción, teoría, práctica y resumen. Los seminarios de esta asignatura tienen un carácter teórico-práctico y es el objeto de la intervención. Se comienza realizando una pequeña introducción y un guion para intentar contextualizar la clase. Seguidamente, con una presentación en PowerPoint, se expone la teoría que introduce los conceptos que después se van a trabajar de forma práctica en los ejercicios propuestos. Los problemas prácticos que se incluyen dan respuesta a las preguntas recogidas en el mapa de contenidos. Las prácticas de la asignatura constan de una breve introducción y realización de unas prácticas de laboratorio siguiendo un guion, dichas prácticas se

Jornadas de Formación e Innovación Docente del Profesorado I № 1 (2018)
Esta obra se distribuye con la licencia Creative Commons Reconocimiento-NoComercial-SinObraDerivada $\quad 4.0$ Internacional (CC BY-NC-ND 4.0.) 
basan en la determinación analítica de diferentes parámetros de interés en los alimentos y esatn relacionadas con la pregunta 4 del mapa de contenidos anteriormente expuesto. El carácter procedimental está muy marcado en el caso de la determinación y de la elaboración de las tablas de composición. Se intenta relacionar los contenidos del seminario con asuntos que ya se han visto o que se verán después para que no de la sensación de conceptos aislados y mediante pequeñas preguntas se intenta captar su atención. En este aspecto hay veces que hay mucha participación y en otro caso largas esperas. Al pasar a la parte de resolución de ejercicios normalmente son los estudiantes los que corrigen, dialogan y resuelven estas cuestiones. En este aspecto el ambiente del grupo favorece o no la participación de los alumnos y la forma de resolver las dudas. Si el ambiente es distendido todo se realiza con mayor naturalidad y con un trato mucho más cercano (Bain, 2007). Finalmente se realiza un resumen de los temas tratados hasta el momento y se proporciona un avance sobre el siguiente seminario y las recomendaciones sobre el material que será necesario, etc. El tiempo que se suele emplear depende del empleado el resto de actividades. La secuencia de actividades en cada una de las sesiones de seminarios es la que a continuación se indica:

1. Breve introducción de la temática. Contextualización e indagación sobre conocimientos previos (5 minutos).

2. Presentación de los contenidos teóricos iniciales (15 minutos).

3. Resolución de cuestiones relacionadas. Trabajo personal de los alumnos sobre problemas de las cuestiones clave y apoyo del profesor (25 minutos).

4. Reflexión sobre los contenidos. Ideas clave extraídas. (15 minutos).

Los porcentajes aproximados de cada una de las partes son $10,25,40$ y $10 \%$ respectivamente, siendo consecuente con el modelo metodológico. 
Según lo descrito, la modelización de una clase habitual de seminarios y de una sesión de laboratorio tiene un modelo como el que se muestra en la Figura 3. En este modelo se presenta la distribución de los tiempos empleados en cada una de estas actividades.

\section{Modelo metodológico}

Seminarios

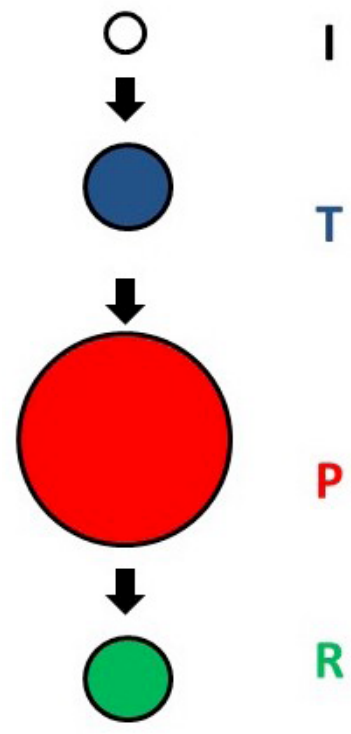

Laboratorio

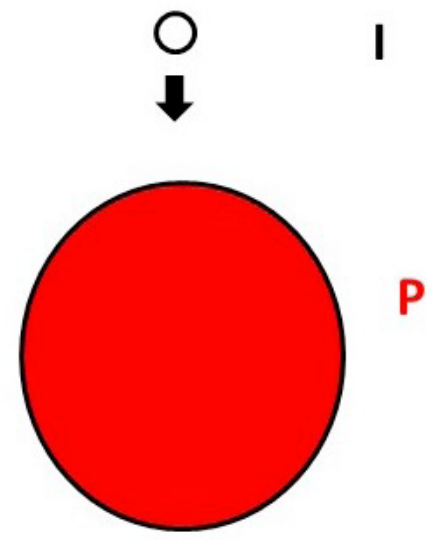

\section{I: introducción; T: teoría; P: practica ; R: resumen}

Figura 3. Modelo metodológico seguido en la docencia de las clases de seminarios y laboratorio de Nutrición y Bromatología.

En el caso de una sesión de laboratorio la división temporal corresponde con un $2 \%$ de introducción y un $98 \%$ de realización de la práctica, como se indicó anteriormente. Muchas veces no se deja tiempo suficiente para el correcto análisis de los datos obtenidos ni para una posterior reflexión sobre la utilidad de la práctica realizada.

A veces el seguimiento de la asignatura resulta bajo y se produce una falta de asistencia a clase. Una de las 
opciones es la utilización de nuevas metodologías docentes que incrementen y favorezcan el seguimiento de las clases y la asignatura por parte del alumnado. Se plantea la gamificación como herramienta para conseguir el fin anteriormente mencionado en el caso de las sesiones de seminarios. En el caso de las sesiones de laboratorio se desea utilizar las preguntas clave para hacer reflexionar sobre la finalidad de dicha actividad y su relación con contenidos que ya debe poseer el alumnado.

\section{Innovación metodológica: introducción de elementos gamificadores en el aula de seminarios. Modelo metodológico modificado}

Se planteó crear una competición a lo largo del curso para tener una clasificación por grupos de seminario de los alumnos que participen de forma voluntaria en un concurso de preguntas y respuestas por medio de Socrative. El objetivo del juego es tener el mayor número de puntos para poder obtener hasta un punto en el examen final de esta parte. El modelo metodológico se vio ligeramente modificado tal como se puede observar en la Figura 4.

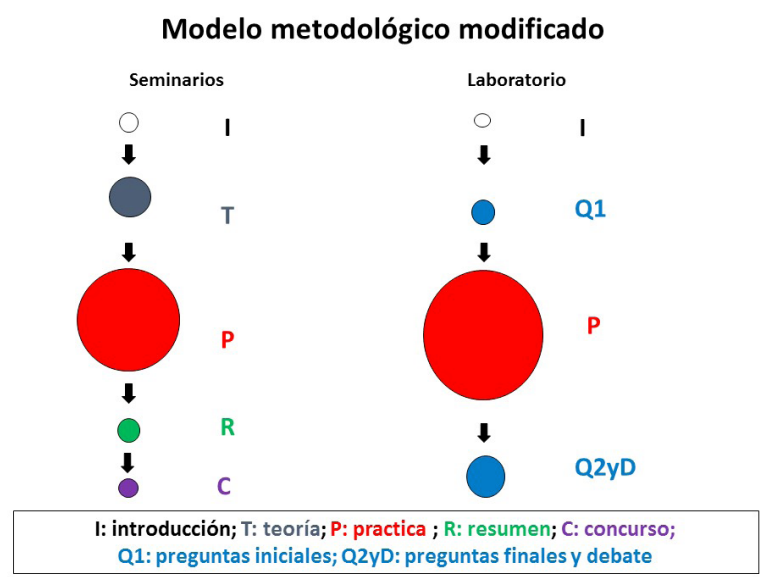

Figura 4. Modelo metodológico modificado seguido en la docencia de las clases de seminarios y laboratorio de Nutrición y Bromatología.

Jornadas de Formación e Innovación Docente del Profesorado | № 1 (2018) 
Como se puede ver de forma gráfica la distribución temporal solamente se ve ligeramente modificada en la parte final. El coste en cuanto a tiempo de clase no es muy elevado y por tanto se estima que puede ser de fácil implantación. Sin embargo el tiempo dedicado a la planificación del juego si requiere un esfuerzo considerable por parte del profesor.

\section{Innovación metodológica: laboratorio de la asignatura Nutrición y Bromatología. Modelo metodológico modificado}

En el caso de las sesiones de laboratorio se pretendió utilizar las preguntas clave. La idea fue leer solamente el título de la práctica y que los alumnos planteasen dos preguntas a las que la práctica da respuesta. Tras su realización se volvería a pedir otra vez las preguntas y se sugeriría posteriormente que mediante un pequeño debate se decidiese si la realización de la práctica ha dado respuesta o no y con qué conceptos básicos estaban relacionadas las respuestas obtenidas. Se incluyó en dicho diseño una parte inicial de cuestionamiento respecto a la realización de la práctica y una parte final de reflexión de la misma quedando la distribución de actividades como se muestra en la Figura 4.

\section{Descripción de la actividad de gamificación en el aula}

A continuación se describe detalladamente el concurso utilizado para la gamificación de los seminarios. Para no someter a alumnado a una competitividad excesiva en vez de un ranking se propone realizar unos cuartiles con los puntos alcanzados en el concurso y repartir hasta un punto para la evaluación de esta parte de la asignatura. El Socrative se realizará de forma individual y cada pregunta acertada suma un punto para la clasificación. La fecha de la prueba tampoco fue avisada. Para los ejercicios 
en los que se requiera colaboración en clase se realizaron subastas de puntos para hacer el ejercicio. Se pretendió con toda esta estrategia una mayor asistencia a clase.

La secuencia de actividades en cada una de las sesiones de seminarios fue:

1. Breve introducción de la temática. Contextualización e indagación sobre conocimientos previos (5 minutos).

2. Presentación de los contenidos teóricos iniciales (15 minutos).

3. Resolución de cuestiones relacionadas. Trabajo personal de los alumnos sobre problemas de las cuestiones clave y apoyo del profesor. Problemas numéricos, búsqueda de información etc. Conceptos específicos del problema no vistos en la presentación inicial de contenidos (25 minutos).

4. Reflexión sobre los contenidos. Ideas clave extraídas. (10 minutos).

5. Concurso (5 minutos).

\section{Valoración de la actividad de gamificación y su utilidad en la asignatura}

El objetivo se estimaría como cumplido si el seguimiento del juego representa un porcentaje alto en la clase sin decaer a lo largo del tiempo. Se evaluó dicha participación en el concurso y los resultados en esta parte de la asignatura por parte de los alumnos participantes con respecto a las calificaciones promedio obtenidas en otros cursos o incluso con las de compañeros que no han querido participar en la actividad serán evaluadas cuando se disponga de datos de la evaluación intermedia o parcial y de la evaluación final. Al final también se realizó un pequeño cuestionario de opinión para conocer como han valorado la actividad propuesta. 
Seguimiento de la evolución de los modelos mentales de los estudiantes

Se pretendió realizar un cuestionario a través de Google Formularios con un total de unas 7 preguntas basadas en las 5 preguntas clave del mapa de contenidos, al inicio de la asignatura y una vez que finalice el ciclo de mejora. Dicho cuestionario fue anónimo siendo el objetivo del mismo ver la evolución del grupo en la escalera en forma de porcentaje de alumnos en cada escalón de la misma. En la propuesta realizada en los seminarios el año anterior no se realizó dicho seguimiento por lo que resultaba de interés hacerlo.

\section{Descripción de la actividad de mejora en el laboratorio}

Se solicitó al inicio de la sesión que solamente con leer el título de la práctica se formulasen dos preguntas a las que la práctica daba respuesta. Dichas preguntas se pidieron por escrito. Tras la realización de la práctica se volvió a pedir las preguntas de nuevo por escrito. Se realizó un pequeño debate y se solicitó nuevamente por escrito la realización de un mapa de contenidos con las preguntas propuestas como interrogantes a los que responde la práctica así como lo contenidos relacionados con las mismas.

La secuencia de actividades en cada una de las sesiones de laboratorio fue la que a continuación se indica:

1. Breve introducción de cuestiones formales de la práctica de laboratorio (seguridad, etc.) y lectura del título de la misma (5 minutos).

2. Petición de preguntas iniciales a las que responde la práctica de laboratorio (5 minutos).

3. Realización de la práctica de laboratorio (2 horas 30 minutos).

4. Petición de preguntas finales, debate, mapa y cuestionario (20 minutos). 


\section{Valoración de la actividad de mejora en el laboratorio}

Al final también se realizó un pequeño cuestionario de opinión para conocer como han valorado la actividad propuesta. La valoración de la actividad se fue realizada por cuestionarios de escala. Dicho cuestionario incluían tres cuestiones. Las dos primeras con dos opciones de respuesta. Sí/No y las preguntas eran las siguientes: ¿Te ha ayudado este ejercicio a ver la aplicación real de la practica realizada?. 2. ¿Te ha resultado interesante y te ha ayudado a aclarar conceptos la discusión con tus compañeros?. La tercera fue una cuestión sobre la valoración global de la actividad, donde los alumnos podían elegir un valor numérico entre 0 y 10, donde 0 corresponde al valor mínimo y 10 al máximo.

\section{Seguimiento de la evolución de los modelos mentales de los estudiantes}

Se tuvo en cuenta la complejidad de las cuestiones iniciales y finales así como la elaboración de pequeños mapas conceptuales relativos a la práctica en cuestión. De esta manera se elaboraron escaleras cuyo objetivo era ver la evolución del grupo en forma de porcentaje de alumnos en cada escalón de la misma (Porlán, 2017).

Jornadas de Formación e Innovación Docente del Profesorado I № 1 (2018) Esta obra se distribuye con la licencia Creative Commons 


\section{Aplicación del ciclo de mejora docente}

\section{Relato resumido de las sesiones y evaluación del aprendizaje de los estudiantes}

\section{Actividad de gamificación en el aula}

A pesar de tratarse de una actividad ya ensayada en el curso pasado y con muy buenos resultados (Hernández Hierro, 2017) este año el escenario ha sido muy diferente. El año pasado los grupos en los que impartía docencia eran grupos de mañana y este año se trataba del último grupo de tarde. En este grupo suelen estar un número elevado de alumnos repetidores que apenas asisten a clase. Hay muy pocos alumnos que asisten con regularidad y el número ha ido bajando de forma gradual al abandonar la asignatura. A pesar de todo he intentado seguir con la propuesta y he seguido la secuencia de actividades. Al presentar la propuesta de gamificación a los alumnos que asisten con regularidad ha sido bien aceptada y han participado, si bien es cierto que son un número reducido. Comentaban que se está aplicando en muchas asignaturas y que están saturados del mismo tipo de actividad, que ciertamente casi resulta para ellos rutinaria. Este año la actividad requería mucho menos trabajo por su parte pues se había eliminado la entrega de posibles preguntas pero a pesar de esto no ha tenido el éxito esperado. Al suceder esto me he sentido algo desmotivado al ver que el objetivo inicial no se cumpliría según lo planificado inicialmente.

Según se indicó el objetivo era conseguir una elevada participación y seguimiento de las clases utilizado estas herramientas para conseguirlo. Teniendo en cuenta el porcentaje de alumnos que asisten a clase de forma regular el objetivo se podría estimar como bueno. Este dato no representa, sin embrago, la situación real pues si consideramos 
el número de alumnos matriculados el porcentaje es muy reducido y ha ido descendiendo. Analizando las pruebas de Socrative podemos observar que los participantes han adquirido bastante bien los conocimientos trabajados dentro de esta parte de la asignatura. En este sentido creo que dichos alumnos trabajarían los contenidos de cualquier otra forma sin problemas y son del $15 \%$ que son prácticamente independientes de la metodología aplicada (Jiménez Font, 2017).

Se envió un cuestionario a través de Google Formularios con un total de unas 7 preguntas basadas en las 5 preguntas organizadoras o clave del mapa de contenidos. En este sentido cabe destacar que en la propuesta inicial se obtuvieron un número de respuestas como para hacer una escalera de aprendizaje de tres niveles aproximadamente situándose en torno a un 30\% en el escalón inicial, 60\% en el intermedio y el restante $10 \%$ en el escalón con un grado de complejidad más elevado. No ha sido posible, o al menos no se ha considerado pertinente por su baja significación a nivel poblacional, la realización de la escalera final y por consiguiente la comparación de dichas escaleras al encontrarse solamente dos respuestas en la encuesta final.

\section{Actividad de mejora en el laboratorio}

Antes de empezar la práctica las preguntas efectuadas no corresponden, o lo hacen vagamente, a las que daría respuesta la práctica. En numerosa ocasiones dichas preguntas se centraban mucho más en el detalle que en la cuestión general que estaba resolviendo. Al comenzar realizando la práctica con la pregunta de su utilidad están reflexionando sobre esa cuestión durante todo el tiempo de realizacion práctica. Esto resulta beneficioso pues lo hacen incluso en los tiempos muertos que tienen las prácticas, ayudando a conservar un buen ambiente de trabajo en el laboratorio. Después de realizar la práctica se observó que las preguntas tenian un grado de complejidad 
mucho mayor y se ajustaban al objetivo de la práctica. En el pequeño debate hay muchas aclaraciones, algunas veces volviendo de nuevo al detalle y perdiendo un poco el objetivo general buscado. Elaboraron diferentes mapas de contenidos con una variabilidad de representación que es elevada, si bien todos ellos dierón una buena idea de la práctica realizada. En general, el ambiente distendido contribuyó a que toda la actividad se puediese realizar con tranquilidad y prestando atencion a lo que se estaba haciendo.

Al final también se realizó un pequeño cuestionario de opinión con las siguientes preguntas: ¿Te ha ayudado este ejercicio a ver la aplicación real de la practica realizada?. 2. ¿Te ha resultado interesante y te ha ayudado a aclarar conceptos la discusión con tus compañeros?. La tercera sobre la valoración global de la actividad, donde los alumnos podrán elegir un valor numérico entre 0 y 10, donde 0 corresponde al valor mínimo y 10 al máximo.

El porcentaje de respuestas positivas recibidas en la primera y en la segunda respuesta fue en torno a un $90 \%$, lo que muestra una buena aceptación de esta actividad en las prácticas. En lo referente a la tercera pregunta, la nota obtenida fue de 8.2, lo que también indica buena valoración de la misma.

Si analizamos las preguntas iniciales y finales en forma de escalera de conocimiento y a tres niveles en el caso inicial la distribución fue de $60 \%, 30 \%$ y $10 \%$ en los niveles bajo, medio y alto respetivamente. Estos porcentajes cambiaron de forma drástica al final de la realización de la misma siendo $5 \%, 70 \%$ y $25 \%$ en los niveles bajo, medio y alto respetivamente.

Al construir los mapas de contenidos de forma conjunta también se puede corroborar la información obtenida en ese cuestionario final. Se puede comprobar que el grado de complejidad de estos mapas coincide con los datos arriba mencionados, estando por lo tanto en un nivel adecuado medio-alto de asimilación de los contenidos tratados. 


\section{Evaluación del ciclo de mejora docente}

\section{Cuestiones a mantener y cambios a introducir}

Teniendo en cuenta los resultados obtenidos en la implementación del ciclo de mejora de laboratorio creo conveniente mantener la secuencia de actividades que se ha propuesto en este ciclo. Estas cuestiones activan la curiosidad del alumno y lo tienen activo durante la realización de la práctica. Respecto a la actividad de gamificación en los seminarios, y pese a los malos resultados, creo conveniente mantenerla. El ambiente y los alumnos son diferentes cada año y no encuentro la razón para que un año en el que la actividad estaba más trabajada haya funcionado peor. Creo que también es importante mostrar que no siempre las cosas salen como idealmente queremos y que al trabajar con un factor humano es muy dificil controlar ciertos aspectos. Sin embargo todos estos obstáculos no deben frenar nuestras ideas de cambio para la mejora docente. El proceso es largo y es conveniente poner a prueba algunas cosas varias veces antes de desecharlas. Otra cuestión a destacar es la forma de plantear el ciclo de mejora, en esta ocasión y por la experiencia a de la vez anterior (Hernández Hierro, 2017) el ciclo fue planteado como voluntario, sin embargo en futuras ediciones, el eventual ciclo de mejora será aplicado a la totalidad de los alumnos. En este sentido también cabe destacar que los futuros ciclos prioritariamente irán encaminados en la mejora de las actividades procurando que el trabajo con los problemas del mapa de contenidos sea el elemento motivador.

\section{Aspectos de la experiencia que se pretenden incorporar a toda la práctica docente habitual}

Como he indicado anteriormente se pretende mantener la secuencia de actividades de la práctica tal y como 
ha sido propuesta en este curso. Es una buena estrategia y la relación esfuerzo-beneficio es muy alta. Con lo que respecta a la parte de los seminarios se pretende mantener la parte de la gamificación, el curso anterior (Hernández Hierro, 2017) proporcionó buenos resultados y es necesario contrastar de nuevo dicha actividad para reafirmarme en su utilidad o por el contrario tomar la decisión de eliminarla. En futuras ocasiones no plantaré como opcional esta actividad. Además de instaurar la actividad de gamificación, en mi práctica docente el planteamiento de trabajo con los problemas del mapa de contenidos deberá de ser el eje central.

\section{Principios didácticos argumentados}

No tener miedo a equivocarse y en caso afirmativo tener capacidad para rectificar y asumir la equivocación, pero no abandonar.

Tener claro que hay un sinfin de factores que no podemos controlar como docentes y por tanto que tenemos que asumirlos.

El factor humano es uno de los más importantes y aunque pueda seguir unos patrones más o menos comunes es difícil de controlar. Intentar facilitar y hacer interesantes los contenidos de manera que sea más sencillo y ameno su aprendizaje.

Jornadas de Formación e Innovación Docente del Profesorado | № 1 (2018) Esta obra se distribuye con la licencia Creative Commons 


\section{Referencias bibliográficas}

AulaPlaneta (2015). Cómo aplicar el aprendizaje basado en juegos en el aula [en línea] Disponible en: http:// www.aulaplaneta.com/2015/08/11/recursos-tic/ como-aplicar-la-gamificacion-en-el-aula-infografial

Bain, K. (2007). Lo que hacen los mejores profesores universitarios. Universidad de Valencia: Publicaciones de la Universidad de Valencia.

Decanato de la Facultad de Farmacia de Sevilla (2017). Guía de la Facultad de Farmacia curso 2017/2018. Recuperado de http:// www.farmacia.us.es/guia.

Hernández Hierro, J. M. Uso de elementos de gamificación en los seminarios de la asignatura nutrición y bromatología. IV Jornadas de formación e innovación docente Universidad de Sevilla, pág. 875. (2017).

Jiménez Font, $X$. ¿Por qué y cómo cambiar la docencia universitaria? IV Jornadas de formación e innovación docente Universidad de Sevilla. (2017).

Porlán, R. (2017). Enseñanza universitaria. Cómo mejorarla. Universidad de Sevilla: Ediciones Morata, S. L.

Jornadas de Formación e Innovación Docente del Profesorado | № 1 (2018) Esta obra se distribuye con la licencia Creative Commons 